\title{
Cylindric Electron Envelope for Relativistic Electron Cooling
}

\author{
A. Burov, V. Lebedev
}

March 18, 2005

\begin{abstract}
Electron cooling requires small angles between the electrons and the cooled particles. In other words, the electron beam has to be of a cylindrical shape in the cooling section. How to satisfy this requirement for a specific case of a relativistic electron beam transport for the Fermilab electron cooling project? In fact, the requirement splits into two parts: for the beam centroid and its envelope. A straight centroid motion means a good field quality and zero initial conditions; this issue is not a subject of this paper. The cylindrical envelope requires proper initial conditions of the envelope at the entrance of the cooler, it is a problem of matching. A specific complex of measurements and calculations aimed at solving this problem is described here.
\end{abstract}

\section{Introduction}

Electron cooling of the pbars in the Recycler [1] is supposed to increase several times the luminosity of the Tevatron complex. One of the key issues for electron cooling in general and for the Fermilab project in particular is alignment: the angles between electrons and pbars in the cooler have to be limited by 2-3 r. $\mathrm{m}$. s. angles of pbars, or by 100-200 $\mu \mathrm{rad}$, depending on the pbar emittance [2]. To minimize the angles, the electron beam has to be cylindric in the cooler, which means that both its centroid and envelope oscillations have to be small enough. Suppression of the centroid motion requires, first of all, a good field quality in the cooler; this issue is discussed in Ref. [3]. Suppression of the envelope angles requires a specific complex of measurements and analysis, which is a main subject of this paper.

A necessary condition for getting a cylindric beam in the cooler is matching of the magnetic fluxes at the gun and in the cooler:

$$
B_{c}^{2} r_{c}^{4}=B_{g}^{2} r_{g}^{4}+4 K(B \rho)^{2} r_{c}^{2} \equiv \Phi^{2}+4 K(B \rho)^{2} r_{c}^{2} .
$$

Here $B_{c}$ and $r_{c}$ are the magnetic field and the beam radius in the cooler, $B_{g}$ and $r_{g}$ are the same values at the gun, $B \rho \equiv p_{0} c / e$ is the beam momentum, and

$$
K=\frac{2 I e}{m c^{3} \gamma^{3} \beta^{3}}
$$

is the generalized perveance (see, e. g. [4]). Note that the considered electron beam is hydrodynamic, in other words, its temperature, associated with the cathode temperature, can be neglected. The electron beam radius in the cooler $r_{c}$ is determined by the pbar beam radius: for optimal conditions the electron radius is 2-3 times larger than the pbar one, which leads to a number $\sim 3-5 \mathrm{~mm}$. Magnetic field in the cooler has to be strong enough to provide sufficient focusing; 100-200 G is supposed to be sufficient. The beam radius and the field determine the magnetic flux $\Phi$, associated with emittance of the angular-momentum-dominated beam [5], [6]:

$$
\varepsilon=\Phi /(B \rho) ; \quad B \rho \equiv p_{0} c / e .
$$

In general, this sort of beams is described by totally coupled optical functions [6]: 


$$
\mathbf{x}(s)=\mathbf{M}(0, s) \operatorname{Re}[\sqrt{\varepsilon} \mathbf{v}(0) \exp (-i \psi)]=\operatorname{Re}[\sqrt{\varepsilon} \mathbf{v}(s) \exp (-i \psi-i \mu(s))],
$$

where $\mathbf{x}=\left(x, x^{\prime}, y, y^{\prime}\right)^{T}$ is a vector describing a point in the beam surface, $\mathbf{M}(0, s)$ is a transfer matrix, $\mu(s)$ is a phase advance between a starting point $s=0$ and the given point $s, \psi$ is a phase, specifying a position on the envelope,

$$
\mathbf{v}=\left(\sqrt{\beta_{x}},-\frac{i(1-u)+\alpha_{x}}{\sqrt{\beta_{x}}}, \sqrt{\beta_{y}} e^{i \nu},-\frac{i u+\alpha_{y}}{\sqrt{\beta_{y}}} e^{i \nu}\right)
$$

is a basis vector,

$$
\mathbf{v}(s)=\exp (i \mu(s)) \mathbf{M}(0, s) \mathbf{v}(0)
$$

and $\beta_{x, y}, \alpha_{x, y}, u, \nu$ are 6 optic functions determined by the envelope initial conditions and the transfer matrix. The emittance and the 6 optic functions determine all local envelope parameters. When these parameters are known at a specific point $s$, their values at any other point $s^{\prime}$ is given by the transfer matrix $\mathbf{M}\left(s, s^{\prime}\right)$.

Below, a procedure of measurement and re-setting to the design value of the beam envelope is proposed. In brief, it consists of the following consequent parts:

- Measurements of the beam cross-section parameters at positions of several scrapers located in the cooling solenoid;

- Calculation of all the six optic functions $\beta_{x, y}, \alpha_{x, y}, u, \nu$ and the emittance $\varepsilon$ at the entrance of the cooler;

- Calculation of a response matrix of the optic functions to supply currents of 6 independent optical elements;

- Calculation of new settings for the 6 supply currents, which provide design values for the optical functions;

- Final cross-section measurements for the new settings. Ideally, the beam envelope has to be cylindric after that.

All the mentioned calculations are based on the cross-section measurements and on an optical model of the electron line. The optical model, in its turn, is supposed to be already verified and corrected by means of analysis of the beam centroid response.

\section{Beam Cross-Sections}

Envelope parameters in the cooling section can be measured by means of scrapers. In the Fermilab prototype line, these measurements were done for 5 scrapers installed inside of $20 \mathrm{~m}$ long solenoid; every scraper was a metallic plate with a round aperture of radius $R_{s}=7.5 \mathrm{~mm}$. The beam, which has sharp boundaries, can be moved transversely in a vicinity of any scraper until it starts touching it. The beam can be moved in various directions, and every direction $k$ gives a pair of numbers $\left(x_{k}, y_{k}\right)$ for the beam centroid at the scraper, associated with touching at this direction. So, a number of known values is equal to the number of directions $N_{d}$ where the beam was moved. In general, the beam cross-section is an ellipse with an arbitrary tilt; this ellipse is described by 3 parameters. The scraper center $\vec{r}_{s}=\left(x_{s}, y_{s}\right)$ is characterized by 2 more unknowns; thus, there are 5 unknowns in total. So, the number of directions has to be more than $5, N_{d} \geq 5$, to get axis and tilt of the beam ellipse at position of the given scraper.

The ellipse point $\vec{r}(\psi)=(x(\psi), y(\psi))$ can be parametrized as follows: 


$$
x(\psi)=a_{x} \cos \psi+x_{c} ; \quad y(\psi)=a_{y} \cos (\psi-\nu)+y_{c},
$$

where $\left(x_{c}, y_{c}\right)$ specifies the measured position of the beam centroid, when the beam surface touches the scraper, and $\psi$ is a running parameter. Compared with Eqs. $(3,4)$, it is found that

$$
a_{x, y}=\sqrt{\varepsilon \beta_{x, y}}
$$

and $\nu$ is the same $x-y$ phase shift as stays in the basis vector (4). The 5 unknowns now are $\left\{a_{x}, a_{y}, \nu, x_{s}, y_{s}\right\}$, while the data is represented by a $2 \times N_{d}$ matrix of the beam centroid positions $\left(x_{c}, y_{c}\right)$. When the beam is moved in the direction $k$ and touches the aperture of radius $R_{s}$ at the tangent azimuth $\psi_{k}$, it can be expressed as

$$
\left(\vec{r}\left(\psi_{k}\right)-\vec{r}_{s}\right)^{2}=R_{s}^{2} ; \frac{d}{d \psi_{k}}\left(\vec{r}\left(\psi_{k}\right)-\vec{r}_{s}\right)^{2}=0
$$

Thus, there are $2 N_{d}$ equations with 5 unknowns $\left\{a_{x}, a_{y}, \widetilde{\nu}, x_{s}, y_{s}\right\}$ and $N_{d}$ unknown tangent azimuths $\psi_{k}$; from here, again, it is seen that at least 5 directions have to be explored. To estimate errors of this analysis, more than 5 directions have to be used. At the Fermilab prototype line, 8 directions were explored; normally they more or less equidistantly cover the entire $2 \pi$. For every scraper position, $2 N_{d}$ equations (8) are equivalent to minimizing a goal function

$$
G=\sum_{k=1}^{N_{d}}\left[\left(\left(\vec{r}\left(\psi_{k}\right)-\vec{r}_{s}\right)^{2}-R_{s}^{2}\right)^{2}+\left(\frac{d}{d \psi_{k}}\left(\vec{r}\left(\psi_{k}\right)-\vec{r}_{s}\right)^{2}\right)^{2}\right] .
$$

From here, the beam cross-section parameters $\left\{a_{x}, a_{y}, \widetilde{\nu}\right\}$ are found at every explored scraper. The errors are found according a conventional formula

$$
\overline{\delta a_{i} \delta a_{j}}=\frac{2 G}{N_{e q}-N_{p a r}} \mathbf{D}_{i, j}^{-1}, \quad \text { with } \quad \mathbf{D}_{p, q} \equiv \frac{\partial^{2} G}{\partial a_{p} \partial a_{q}},
$$

where $N_{e q}=2 N_{d}$ is the total number of equations and $N_{p a r}=5+N_{d}$ is the total number of the unknown parameters.

\section{Optic Functions}

When the beam cross-section parameters $\left\{a_{x}, a_{y}, \widetilde{\nu}\right\}$ are known at sufficient number of positions along the solenoid, the optic functions $\beta_{x, y}, \alpha_{x, y}, u, \nu$ can be calculated in any place there. According to the design, the beam envelope has to be cylindric in the cooling solenoid, which means

$$
\beta_{x, y}=\beta_{0} \equiv B \rho / B_{c} ; \alpha_{x, y}=0 ; u=0.5 ; \nu= \pm \pi / 2
$$

in any point inside the cooling solenoid; the sign of $\nu$ coincides with a sign of the magnetic field $B_{c}$. In the reality, the optic functions differ from their design values, and this is to be corrected. To calculate the optic functions from the cross-section parameters, the solenoid transfer matrix has to be used:

$$
\mathbf{M}\left(s, s^{\prime}\right)=\left(\begin{array}{cccc}
\cos ^{2}(\phi / 2) & \beta_{0} \sin \phi & -\frac{\sin \phi}{2} & -2 \beta_{0} \sin ^{2}(\phi / 2) \\
-\frac{\sin \phi}{4 \beta_{0}} & \cos ^{2}(\phi / 2) & \frac{\sin ^{2}(\phi / 2)}{2 \beta_{0}} & -\frac{\sin \phi}{2} \\
\frac{\sin \phi}{2} & 2 \beta_{0} \sin ^{2}(\phi / 2) & \cos ^{2}(\phi / 2) & \beta_{0} \sin \phi \\
-\frac{\sin ^{2}(\phi / 2)}{2 \beta_{0}} & \frac{\sin \phi}{2} & -\frac{\sin \phi}{4 \beta_{0}} & \cos ^{2}(\phi / 2)
\end{array}\right),
$$

where $\phi=\left(s^{\prime}-s\right) / \beta_{0}$. The solenoid matrix can be presented as a product of a rotation matrix by angle $\phi / 2$ and a rotation-invariant focusing: 


$$
\mathbf{M}\left(s, s^{\prime}\right)=\left(\begin{array}{cccc}
\cos \left(\frac{\phi}{2}\right) & 0 & -\sin \left(\frac{\phi}{2}\right) & 0 \\
0 & \cos \left(\frac{\phi}{2}\right) & 0 & -\sin \left(\frac{\phi}{2}\right) \\
\sin \left(\frac{\phi}{2}\right) & 0 & \cos \left(\frac{\phi}{2}\right) & 0 \\
0 & \sin \left(\frac{\phi}{2}\right) & 0 & \cos \left(\frac{\phi}{2}\right)
\end{array}\right) \times\left(\begin{array}{cccc}
\cos \left(\frac{\phi}{2}\right) & 2 \beta_{0} \sin \left(\frac{\phi}{2}\right) & 0 & 0 \\
-\frac{\sin \left(\frac{\phi}{2}\right)}{2 \beta_{0}} & \cos \left(\frac{\phi}{2}\right) & 0 & 0 \\
0 & 0 & \cos \left(\frac{\phi}{2}\right) & 2 \beta_{0} \sin \left(\frac{\phi}{2}\right) \\
0 & 0 & -\frac{\sin \left(\frac{\phi}{2}\right)}{2 \beta_{0}} & \cos \left(\frac{\phi}{2}\right)
\end{array}\right)
$$

Let the optic functions at the first scraper be chosen for unknowns to be found. Together with the emittance, this gives 7 unknown values. The optic functions at any positions are expressed through the initial optic functions, see Eq. (5). From another side, the total number of equations is $3 N_{s}$ : three ellipse parameters are known at every scraper position, and these parameters relate to the optic functions according to Eqs. (7). Number of explored scrapers has to be sufficient for the number of equations to exceed the number of unknowns, $3 N_{s}>7$ or $N_{s} \geq 3$.

\section{Optics Correction}

After measuring the cross-sections and calculating the optic functions, they are seen as different from their design values. The entire problem grows from the fact that the model of the beam optics is not good enough. At best, it is only approximately correct, and the errors are expected to be higher than the tolerances. Let it be assumed, first, that there is no reliable optic model at all. Could the designed cylindric envelope be yet established in this case?

Let $\mathbb{F}=\left(\beta_{x} / \beta_{0}, \alpha_{x}, \beta_{y} / \beta_{0}, \alpha_{y}, u / 0.5,2 \nu / \pi\right)^{T}$ be a $6 \mathrm{D}$ vector of the found optic functions at the first scraper, $\mathbb{F}_{0}=(1,0,1,0,1,1)^{T}$ be its design value, and $d \mathbb{F} \equiv \mathbb{F}-\mathbb{F}_{0}$. Let it be assumed now that there are 6 "linear independent" optic elements upstream the solenoid; the field magnitude in every one of them is proportional to its supply current, so that the $6 \mathrm{D}$ vector $\mathbb{I}$ describes all of them. A term "linear independent" means here that an optic functions response matrix

$$
\widehat{\mathbb{R}} \equiv \partial \mathbb{F} / \partial \mathbb{I}
$$

is not degenerate, $|\widehat{\mathbb{R}}| \neq 0$. The response matrix $\widehat{\mathbb{R}}$ can be found in the same way, as the optic functions were: it just requires to repeat the entire sequence of the envelope measurements and calculations 6 more times. Let it be assumed now that this matrix is measured. If deviations of the optic functions from their design values are small enough, a change of the currents

$$
d \mathbb{I}=-\widehat{\mathbb{R}}^{-1} \cdot d \mathbb{F}
$$

establishes the design values of the optic functions. In practice, however, the deviations of the optic functions might be not so small. If so, the linear correction (14) should be expected to bring us just closer to the design values, not necessarily right on them. If so, then the functions have to be measured one more time, and one more correction (14) applied. After some number of iterations, the design values should be established. Note that during these iterations the response matrix $\widehat{\mathbb{R}}$ is not required to be measured as many times as the functions $\mathbb{F}$; normally, it should be sufficient to measure it once or twice.

The described procedure should work, but it has a significant practical drawback. Namely, the entire set of cross-section measurements has to be multiply repeated here, which would take significant time. The problem is exacerbated by the fact that these electron measurements are not compatible with the pbars: any pbars circulating in the storage ring (Recycler) would die on the narrow aperture of the scrapers. Remember now that this procedure does not use any model of the beam line optics: all that is needed is taken from the measurements right away. Actually, this is not necessary and can be improved.

Indeed, envelope simulations require two things: first, the "initial conditions" of the basis vector (4), or its value somewhere upstream of all the six tuning optical elements, and rather good knowledge of the 
transfer matrices from these optical elements to the first scraper. If these objects are known, the response matrix $\widehat{\mathbb{R}}$ can be found from the model, without any dedicated measurements. The important point here is that the described iteration procedure does not require extremely good knowledge of this matrix: the procedure converges to exact design values for any matrix, provided that it is not too bad; in the last case, the procedure does not converge at all. An advantage of this way is that modeling of the transfer matrices requires beam centroid measurements, which are compatible with the pbars and have to be done anyway. Practically, the procedure can be organized in the following way, where the scraper measurement data are used as an input for a complex of a specific calculation code (Mathematica ${ }^{\circledR}$, Java or $\mathrm{C}++$ ) and the optical model, implemented in OptiM [7].

- From the scraper measurements, the optic functions at the first scraper are calculated, as described.

- These optic functions are back-propagated by the OptiM code at its starting point, giving initial conditions for the optic functions.

- For these initial conditions, the response matrix $\widehat{\mathbb{R}}$ is calculated by the OptiM model.

- The described sequence of iterations is generated with the OptiM, converging to new optical settings for the currents $\mathbb{I}$.

- The currents in the 6 tuning elements are set in the real beam line now according to the numbers obtained.

- Final scraper measurements are done.

- If the achieved reduction of the envelope deviations is not sufficient, the entire sequence is repeated one more time.

Convergence of this iteration procedure can be improved if to divide it into two stages. At first stage, only the monopole (axially symmetric) envelope perturbation is corrected. For that, only 2 tuning elements are needed, and the two solenoidal lenses right upstream the cooler are the best to use. When the monopole mode is corrected, the entire $6 \mathrm{D}$ correction scheme can be run.

This procedure is supposed to be used as a method to get good envelope quality of electron beam for electron cooling of pbars in the Recycler. A simplified monopole-only correction scheme, close to the one described, has been successfully applied for the prototype line right before dismantling it [8]. The entire $6 \mathrm{D}$ procedure was tested for these scraper measurements in the prototype line, and good convergence in Mathematica ${ }^{\circledR}$ \& OptiM simulations to the design parameters was seen. Fig. (1) shows cross section projections $a_{x, y}$ calculated with their error bars from the scraper measurements, seen reasonably close to the best fit of the beam envelope in the cooling section.

\section{References}

[1] J. Leibfritz et al., "Status of the Fermilab Electron Cooling Project", Proc. EPAC 2004, p. 1487 (2004).

[2] A. Burov, "Antiproton Stacking in the Recycler", Fermilab-Conf-03-171

[3] V. Tupikov, S. Nagaitsev, A. Shemyakin, S. Seletskij, "Toward a Cold Electron Beam in the Fermilab Electron Coller", Fermilab-Conf-04-058-AD, 2004.

[4] M. Reiser, "Theory and Design of Charged Particle Beams", J. Wiley \& Sons, p. 210 (1994).

[5] A. Burov, S. Nagaitsev, A. Shemyakin, Ya. Derbenev, "Optical principles of beam transport for relativistic electron cooling", Phys. Rev. ST-AB, Vol. 3, 094002

(2000). 


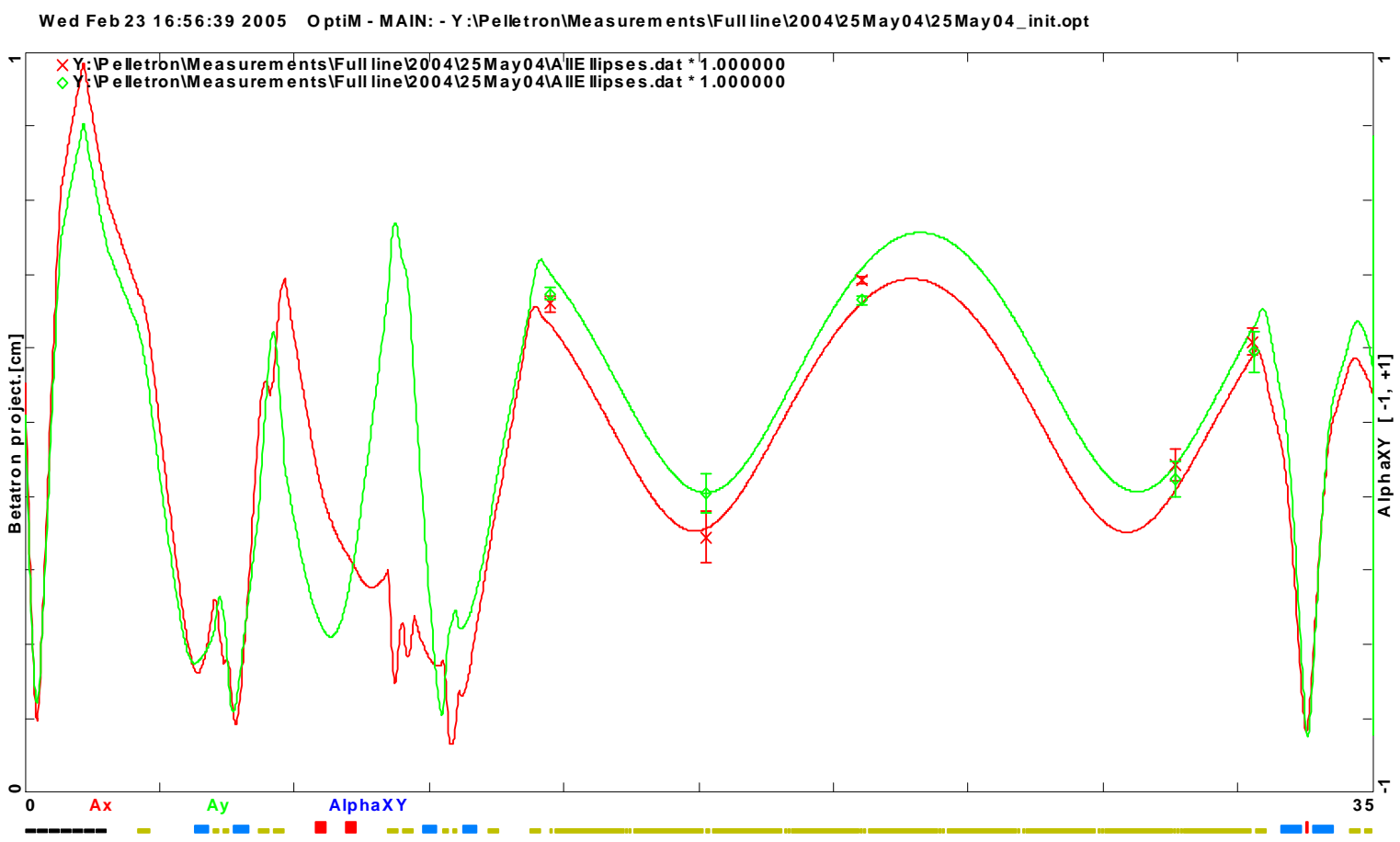

Figure 1: Beam ellipse projections $a_{x}$ (red )and $a_{y}$ (green), calculated from the scraper measurements, and the best fit for the envelope.

[6] V. Lebedev, A. Bogacz, "Betatron motion with coupling of horizontal and vertical degrees of freedom", e-print JLAB-ACC-99-19.

[7] V. Lebedev, http://www-bdnew.fnal.gov/pbar/organizationalchart/lebedev/OptiM/optim.htm .

[8] A. Shemyakin, May 29, 2004. 